\title{
Environmental and Human Impacts of Unconventional Energy Development
}

7 he rise of the "shale revolution" at the beginning of the 121 st century triggered a hotly contested public debate concerning the balance between the economic benefits of extraction of natural gas and oil from unconventional resources (unconventional oil and gas; UO\&G) and the associated environmental and health risks.. This extraction involves first drilling a vertical or horizontal well and then injecting under high pressure millions of gallons of water, sand, and chemicals into the well to induce the formation of fractures in lowpermeability geological formations, allowing for flow of natural gas and/or oil, and returned hydraulic fracturing fluids mixed with formation water to the surface. Benefits are purported to include an expanded energy portfolio and reduced climate forcing through greater availability of natural gas, whose combustion emits less carbon dioxide and other air pollutants than for coal. Risks are purported to include increased climate forcing as a result of fugitive methane emissions, localized air and water pollution, and ecological and community impacts. While the early scientific evidence to inform this debate was slim, the current exponential growth of research in this area is in concert with the expansion of extraction operations, mostly in the U.S. but also in Canada, South America and China. Of the 180 studies published on this topic between 2010 and 2016 in Environmental Science \& Technology (ES\&T) and Environmental Science \& Technology Letters (ES\&T Letters), 75\% were published after 2013 (Figure 1).

Four years ago, the U.S. National Research Council organized workshops in which academic, industry, and

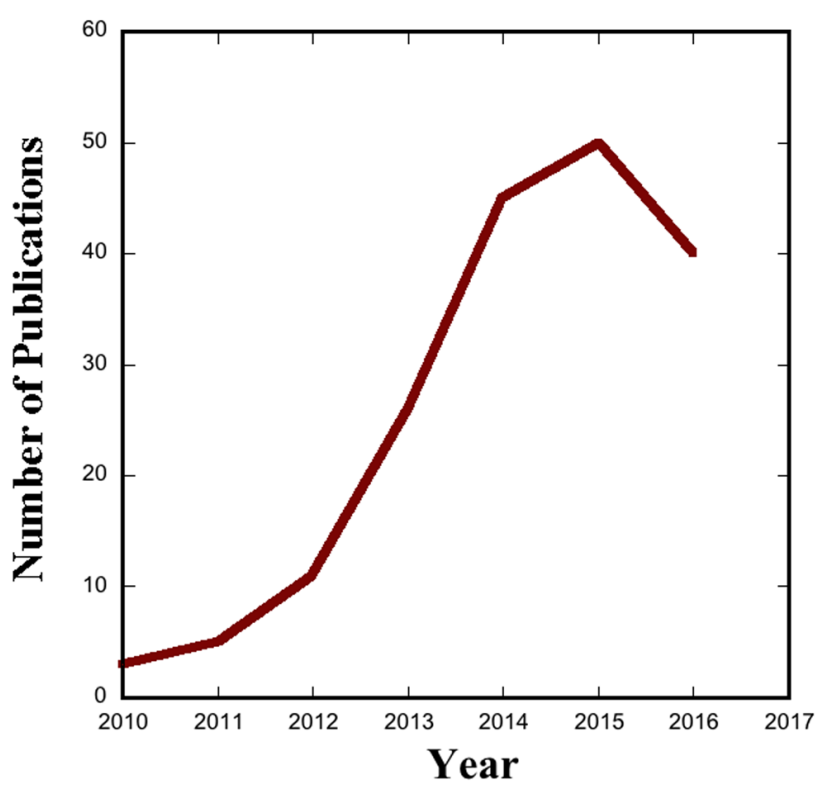

Figure 1. Annual UO\&G-related publications in ES\&T and ES\&T Letters since 2010. Note that the decrease in 2016 reflects an overall decrease in the number of publications in ES\&T and ES\&T Letters. government scientists identified risks associated with UO\&G to (1) water resources; (2) air quality and climate change; (3) public health; and (4) socioeconomic and community effects. An evaluation of these risk domains were published as five reviews in a 2014 ES\&T Special Issue: Understanding the Risks of Unconventional Shale Gas Development. To highlight research advances in more than 100 papers published since that Special Issue, ES\&T and ES\&T Letters have now organized a Virtual Issue containing 28 recent (2015-2017) papers that have contributed to the scientific understanding of UO\&G within the following topics: fugitive methane emission estimates; air quality; impacts of waste fluids (hydraulic fracturing fluids and formation waters) on water quality and use; and risk governance policies.

A 2014 review of UO\&G impacts on global climate change identified that the accurate quantification of fugitive methane emissions from UO\&G is a major priority. "Top-down” studies featured in this Virtual Issue involving aircraft-based methane measurements indicate that methane emissions from Barnett Shale oil and gas operations have been underestimated by a factor of 3 and Marcellus Shale unconventional natural gas well sites have been underestimated by $>10-40$ times. In the Marcellus Shale, UO\&G well sites were estimated to emit 23 times more methane than conventional sites and ethane to methane ratios demonstrated that $\sim 80 \%$ of methane emissions in the Barnett Shale region derived from fossil fuel sources. Methods to apportion methane sources in UO\&G basis have been developed and refined: stable isotope methods, particularly deuterium isotopes, can be used to distinguish biogenic (e.g., landfills) from fossil sources of methane, whereas alkane ratios can be used to distinguish natural gas and oil production sources.

A 2014 review of UO\&G risks in the air quality domain identified tropospheric ozone precursors, priority air pollutants, hazardous air pollutants, and particulate emissions from UO\&G as specific risks to air quality. Five of the studies in this Virtual Issue have advanced understanding of these risks. Source apportionment models applied to measurements of these pollutants in the Barnett and Marcellus Shales provide specific pollutant emission rates from UO\&G. The results indicate that emission rates vary by region, and the rates can be used to model effects on regional health and air quality. Additionally, air pollutant emissions (including methane) from $\mathrm{UO} \& \mathrm{G}$ are highly skewed toward a few high-emitting sources referred to as superemitters. For example, tank vents, hatches, and underperforming emission controls contributed to $>90 \%$ of hydrocarbon emissions in a study of 8000 oil and gas well pads in seven U.S. UO\&G basins. However, the drawbacks of air pollutant emissions from UO\&G production must be weighed against potential benefits of switching among fossil fuel sources for combustion. In particular, switching from coal to natural gas

Published: September 19, 2017 
in central heating facilities and power plants has air quality benefits that may offset pollutant emission from UO\&G. For example, the Chinese city of Urumqi's 2012 switch from coal to natural gas in central heating facilities reduced $<2.5 \mu \mathrm{m}$ particulate matter $\left(\mathrm{PM}_{2.5}\right)$ by $60-75 \%$, and also reduced other pollutants, such as $\mathrm{NO}_{x}$.

A 2014 review of UO\&G risks in the water domain identified water availability, the toxicity of man-made chemicals used for hydraulic fracturing, contaminants in formation waters including halides and naturally occurring radioactive materials (NORM), fugitive emissions of hydrocarbons from leaky wells, subsurface flow of contaminated water, and the high frequency of spills and surface water contamination as major risks. However, some of these may not be as concerning as originally thought. For example, an overview of the water footprint of hydraulic fracturing across U.S. shale plays indicates that the water use for hydraulic fracturing is lower than for other energy extraction methods such as coal and uranium mining. While fugitive emissions of methane and other hydraulic fracturing contaminants into shallow groundwater has been demonstrated, a baseline study conducted prior to UO\&G activities in Quebec detected naturally occurring methane in $80 \%$ of local water wells, particularly in areas near major faults. However, many of the above-mentioned concerns have been further substantiated. Several studies have documented the accumulation of NORM, particularly radium in sludge, soils, and sediment; others have shown the impact of native halides, organic hydraulic fracturing fluid additives (see the following four articles: Biocides in Hydraulic Fracturing Fluids: A Critical Review of Their Usage, Mobility, Degradation, and Toxicity; Indications of Transformation Products from Hydraulic Fracturing Additives in Shale-Gas Wastewater; A Framework for Identifying Organic Compounds of Concern in Hydraulic Fracturing Fluids Based on Their Mobility and Persistence in Groundwater; and Impact to Underground Sources of Drinking Water and Domestic Wells from Production Well Stimulation and Completion Practices in the Pavillion, Wyoming, Field)), and hydrocarbons on ground and surface water. Of particular concern, due to their potential toxicity, is the wide array of halogenated compounds, likely produced by reactions between geogenic hydrocarbons, biocides and/or the native halides. Also, the high level of halides in hydraulic fracturing wastewaters (up to 10-fold seawater) are not removed in common wastewater treatment facilities and so may promote disinfection byproduct formation in downstream drinking water plants. Our understanding of the potential ecotoxicity of these wastewaters is growing: chronic exposure of the water flea, Daphnia magna, to as low as $0.04 \%$ by volume of hydraulic fracturing wastewater reduced reproduction rates, with the effects primarily attributed to the organic constituents.

Finally, the 2014 review of risks from UO\&G in the public health, socioeconomic, and community effect domains identified occupational hazards, community exposure to air pollutants, ground and surface water contamination, nonchemical stressors, overburdened municipal services, upended social and cultural patterns, and volatile economic growth followed by out-migration, declining amenity-led development, and decreased financial investment. Two studies in this Virtual Issue highlight limitations in state-level risk governance structures in which the centralized regulators are distant from oil and gas sites while the state benefits from the oil and gas development. Limitations in risk governance structures may result in economic, rural, participatory, and/or distributive injustices that could increase the vulnerability to health risks for populations living near UO\&G sites.

While our understanding of the environmental and human risks and benefits of UO\&G is rapidly advancing, many gaps remain. Several studies have provided critical information for mitigation measures aimed at reducing air pollutant emissions; however, published air pollutant emission rates in regions other than the Barnett and Marcellus Shales are not available and many air pollutants (e.g., aldehydes and polycyclic aromatic hydrocarbons) likely associated with UO\&G have not been well-characterized. Furthermore, in spite of the rise of studies on quantifying fugitive methane emissions from UO\&G, insights on the overall and long-term impact on global warming and possible mitigation measures are needed. Studies on water impacts should further explore the characterization of organic and inorganic contaminants associated with UO\&G and their interactions; the ecological effects induced by the high intensity of UO\&G drilling and operation; and the long-term effects of aquifer contamination as opposed to immediate impacts by surface water contamination from leaks and spills. It is particularly important to characterize whether synthetic hydraulic fracturing fluid additives or geogenic chemicals and byproducts from formation water are the critical drivers of toxicity in hydraulic fracturing wastewaters; if the former, UO\&G operations would represent fundamentally different risks than conventional oil and gas, while in the latter case, UO\&G would only increase the overall exposure to conventional contaminants although at a larger magnitude given the high intensity of UO\&G development. Future studies should also explore advanced and economically feasible technologies for treatment and reuse of UO\&G wastewater. Currently, most of the UO\&G in the U.S. is disposed through deep-well injection and/or reuse for hydraulic fracturing, but induced seismic activities from deep-well injection in some areas could limit this operation and thus wastewater treatment could become a limiting factor for future UO\&G operations. Many critical research needs remain in the public health and social effects domains, including: determining the magnitude and duration of human exposures to hazardous air and water pollutants emitted from UO\&G; disease surveillance in UO\&G workers and communities; understanding the interaction between chemical and nonchemical (e.g., social, infrastructure) UO\&G stressors; effects of UO\&G related stigma and conflict on long-term community investment and sustainability; and the effectiveness of specific UO\&G risk governance structures. Finally, much of the UO\&G environmental research has been focused thus far on the U.S. case studies; however, the expected rise of global UO\&G development would inevitably expand the international research.

Given this long list of research needs, we invite the community to continue submitting studies to $E S \& T$ and $E S \& T$ Letters that significantly advance our understanding of the environmental and human risks and benefits of UO\&G. Objective scientific research on environmental issues associated with $U O \& G$ is especially needed given the intense public debate on the environmental effects of UO\&G, which has created divisions between industry, environmental groups, and the scientific community. In some cases, these divisions have limited the ability of the scientific community to conduct objective research, mainly due to lack of full accessibility to the research sites and/or actual sampling of hydraulic fracturing chemicals and fluids. Building the bridges and providing the scientific community the ability to conduct independent 
research is critical to the sustainability and future of global $\mathrm{UO} \& \mathrm{G}$ development. Given the contentious nature of this topic, we also challenge the scientific community to develop communication strategies that foster a greater understanding of their findings in the broader public. Dissemination of evidence gained from rigorous scientific studies published in the peer reviewed literature to the public will be critical to counteract the misrepresentation of environmental and health risks and benefits that has been done by special interest groups. While the bulk of the ES\&T and ES\&T Letters publications on $\mathrm{UO} \& \mathrm{G}$ have been research articles, we remind the community of the need and opportunity for publication of UO\&G policy analyses that provide a vehicle to frame policy relevant research results.
Avner Vengosh $*, \dagger$
William A. Mitch ${ }^{\ddagger}$
Lisa M. McKenzie ${ }^{\S}$
${ }^{\dagger}$ Nicholas School of Environment and Earth Science, Duke University, Durham, North Carolina 27708, United States
${ }^{\ddagger}$ Civil and Environmental Engineering, Stanford University, Stanford, California 94305, United States
${ }^{\S}$ Department of Environmental and Occupational Health, Colorado School of Public Health, Aurora, Colorado 80045, United States

\section{AUTHOR INFORMATION}

\section{Corresponding Author}

*E-mail: vengosh@duke.edu.

\section{Notes}

Views expressed in this editorial are those of the authors and not necessarily the views of the ACS.

The authors declare no competing financial interest. 